\title{
HIV protease inhibitors modulate apoptosis signaling in vitro and in vivo
}

\author{
Stacey R. Vlahakis • Steffany A. L. Bennett • \\ Shawn N. Whitehead • Andrew D. Badley
}

Published online: 9 February 2007

(C) Springer Science + Business Media, LLC 2007

\begin{abstract}
HIV protease inhibitors are an integral part of effective anti-HIV therapy. The drugs block HIV protease, prevent proper packaging of HIV virions, and decrease the HIV viral burden in the peripheral blood of infected individuals. In addition to direct anti-viral effects, the HIV protease inhibitors also modulate apoptosis. A growing body of work demonstrates the anti-apoptotic effects of HIV protease inhibitors on $\mathrm{CD}^{+}$and $\mathrm{CD} 8^{+} \mathrm{T}$ cells during HIV infection. The mechanism of this apoptosis inhibition is supported by several proposed hypotheses for how they alter the fate of the cell, including preventing adenine nucleotide translocator pore function, which consequently prevents loss of mitochondrial transmembrane potential. More recently, the anti-apoptotic effects of the HIV protease inhibitors have been tested in non-HIV, non-immune cell, whereby protease inhibitors prevent apoptosis, and disease in animal models of sepsis, hepatitis, pancreatitis and stroke. Interestingly, when HIV protease inhibitors are used at supra-therapeutic concentrations, they exert pro-apoptotic effects. This has been demonstrated in a number of tumor models. Although it is unclear how HIV protease inhibitors can induce apoptosis at increased concentrations, future research will define the targets of the immunomodulation and reveal the full clinical potential of this intriguing class of drugs.
\end{abstract}

S. R. Vlahakis · A. D. Badley $(\triangle)$

Division Infectious Disease, Mayo Clinic College of Medicine,

200 First Street SW, Rochester, MN 55905

e-mail: badley.andrew@mayo.edu

S. A. L. Bennett · S. N. Whitehead

Neural Regeneration Laboratory, Department of Biochemistry,

Microbiology and Immunology, University of Ottawa,

451 Smyth Road, Ottawa, ON, Canada, K1M 1E5
Keywords Apoptosis · HIV protease inhibitors · Neurons · Mitochondria

HIV infection causes a progressive depletion of $\mathrm{CD} 4^{+} \mathrm{T}$ cells. There are likely many reasons for the $\mathrm{CD} 4^{+} \mathrm{T}$ cell loss, including apoptosis. Current therapy for HIV-infected patients often includes a combination of reverse transcriptase inhibitors and HIV protease inhibitors. The first HIV protease inhibitor was FDA approved for anti-HIV therapy in humans in 1995. Currently, there are ten HIV protease inhibitors that are approved for clinical use in humans (saquinavir, indinavir, darunavir, nelfinavir, lopinavir, amprenavir, atazanavir, tipranavir, ritonavir, and fosamprenavir). These drugs are inhibitors of the HIV viral aspartyl protease. The compounds have a strong affinity for the active site of the HIV protease and irreversibly inhibit the catalytic activity of the enzyme.

When HIV protease is inhibited, viral particles are produced, but they are immature, and do not package properly into infectious virions. Moreover, naturally occurring HIV protease mutations, which arise during suboptimal antiretroviral therapy, result in impaired replication kinetics of progeny virions. In addition to its role in viral replication, HIV protease may also contribute to HIV pathogenesis. When transfected into a human or bacterial cell, HIV protease is cytotoxic and causes cleavage of a variety of host proteins including actin, $\mathrm{Bcl}_{2}$ and procaspase 8 . It remains unclear how HIV protease initiates cell death.

Although the HIV protease inhibitors have limited bioavailability and stability, they are the basis of effective anti-HIV therapy, and in combination with other antiretroviral agents, produce a sustained decrease in HIV viral load. Over the past ten years, evidence has accumulated that HIV protease inhibitors have non-viral effects on the host cells beyond the effect of blocking HIV protease enzymatic 
activity. For example, HIV protease inhibitors also directly affect cellular apoptosis signaling.

\section{The role of HIV protease inhibitors during treatment of HIV infection}

Optimal anti-HIV therapy is called highly active antiretroviral therapy (HAART). This includes the combination of HIV reverse transcriptase inhibitors, often with an HIV protease inhibitor. Since the advent of HAART in late 1995, these regimens have been shown to increase $\mathrm{CD} 4^{+} \mathrm{T}$ cell counts and reduce the amount of HIV virus quantitated in the peripheral blood, in infected individuals. However, in many cases, the increase in $\mathrm{CD}^{+} \mathrm{T}$ cells that occurs during HIV therapy appeared to result from actions that were independent of the effect of the drug on viral replication. In one such instance, HIV-infected subjects were randomized in a clinical trial to receive therapy with three drugs, including one protease inhibitor (saquinivir, zidovudine, and zalitabine) or two drugs. One of the two drug regimens included a protease inhibitor (zidovudine and saquinivir), and the other two-drug regimen had no protease inhibitors (zidovudine and zalitabine) [1]. Two-hundred and eighty subjects completed 24 weeks of treatment. Even though there was worse virologic control in the two-drug, than the three-drug regimen group, the subjects in the two-drug group who received the HIV PI had improved $\mathrm{CD}^{+}{ }^{+} \mathrm{T}$ cell counts, compared to the two-drug group that did not receive an HIV PI. Subsequent clinical trials confirmed that HIV protease inhibitors improved CD4 ${ }^{+}$ $\mathrm{T}$ cell counts in HIV-infected individuals, independent of the viral suppression [2-5]. In a meta-analysis comparing antiHIV drug regimens using a PI to those that switched the PI to a non-nucleoside reverse transcriptase inhibitor (NNRTI), PI-based therapy resulted in higher $\mathrm{CD}^{+} \mathrm{T}$ cell counts [6]. The analysis required that both PI and non-PI based regimens have complete HIV viral suppression. Therefore, the $\mathrm{CD} 4^{+} \mathrm{T}$ cell benefit with HIV protease inhibitor therapy appeared to be, in part, unrelated to the effect on HIV viral suppression. In a different trial comparing treatment with HIV protease containing highly active antiretroviral therapy (HAART) to protease inhibitor therapy alone, there was less virologic suppression of the subjects on PI mono-therapy. However, there was no decrease in $\mathrm{CD} 4^{+} \mathrm{T}$ cell counts over one year, even in the group who received PI mono-therapy and virologically failed [7]. Additionally, in a comparison between PI-based anti-HIV therapy to NNRTI-based anti-HIV therapy, there was equal viral suppression in both groups; however, PI-based regimens had a greater increase in $\mathrm{CD} 4^{+}$ $\mathrm{T}$ cell counts [8].

In the absence of effective antiretroviral therapy, HIVinfected subjects have considerable lymphocyte apoptosis in lymphoid tissue. The lymphoid tissue apoptosis is reduced significantly when a patient is treated with HIV PI containing HAART, which correlates with a decrease in HIV peripheral blood viral load and an increase in peripheral blood CD4 ${ }^{+}$ $\mathrm{T}$ cells. In addition, $\mathrm{CD}^{+}$and $\mathrm{CD} 8^{+} \mathrm{T}$ cells isolated from HIV-infected individuals before and on days 1, 4, and 8, after starting treatment with HIV PI containing HAART, had decreased sensitivity to Fas-mediated apoptosis after being on HAART for as little as one day [9].

A recent trial by Landay et al. focused on the mechanism behind HIV protease inhibitor immune effects. The trial compared patients with suppressed HIV viral replication on a PI-based anti-HIV regimen or non-PI based drug regimen. One week after enrollment in the study, subjects in the PI containing arm had less spontaneous T cell apoptosis than those in the non-PI containing arm. Although there are likely multiple reasons for $\mathrm{CD} 4^{+} \mathrm{T}$ cell decline during the course of HIV infection, these data suggest that patients on HIV protease inhibitors have less spontaneous $\mathrm{CD} 4^{+} \mathrm{T}$ cell apoptosis and have improved $\mathrm{CD} 4^{+} \mathrm{T}$ cell counts, further suggesting that the HIV protease inhibitors block $\mathrm{CD} 4^{+} \mathrm{T}$ cell apoptosis, independent of their effect on HIV replication [10].

\section{In vitro anti-apoptotic properties of HIV protease inhibitors}

Due to findings that PI therapy reduced lymphocyte apoptosis, our group proposed in 1998 that highly active antiretroviral therapy (HAART) might independently block cellular apoptosis [9]. Subsequent investigations tested and confirmed this finding ex vivo and in vitro [11, 12]. HIV PIs anti-apoptotic effects were investigated using ritonavir in cultures of bone marrow cells from HIV-infected patients or normal controls. Hematopoietic colony forming unit replication, following addition of ritonavir, had $45 \%$ less apoptosis than untreated cultures. The authors also reported a decrease in caspase-1 expression after ritonavir treatment [13].

In additional experiments investigating the effects of HIV PIs on $\mathrm{T}$ cell death during HIV infection, $\mathrm{CD}^{+}{ }^{+} \mathrm{T}$ cells isolated from healthy uninfected individuals had increased Fas expression and Fas and anti-CD3-induced apoptosis when incubated with HIV virions. Inducible Fas expression and apoptosis were abrogated when the cells were preincubated with the HIV PI, saquinavir [14]. These findings were supported by subsequent investigations which reported that saquinavir and ritonavir inhibited TNF-mediated U937 cell apoptosis in a dose-dependent fashion with $38-60 \%$ reduction in apoptosis in PI treated cells [15]. During HIV infection, the HIV envelope gp120 binds to the CD4 and CXCR4 receptors on the surface of the $\mathrm{CD}_{4}{ }^{+} \mathrm{T}$ cell and signals the cell to undergo apoptosis. This bystander death is one of the ways that HIV depletes the immune system. 
Matarrese et al. treated human $\mathrm{CD}^{+} \mathrm{T}$ cells with HIV gp120 to make the cell sensitive to Fas-mediated apoptosis, which resulted in mitochondrial changes and apoptosis after Fas exposure. When the $\mathrm{CD} 4^{+} \mathrm{T}$ cells were pretreated with a PI before HIV gp120, mitochondrial depolorization was blocked in a whole cell or cell-free system, or isolated mitochondria [16]. Taken together, this data indicates that patients who received HIV protease inhibitors had improved $\mathrm{CD}^{+} \mathrm{T}$ cell counts independent of the state of HIV viral replication, and in vitro work confirmed that HIV PIs can inhibit $\mathrm{T}$ cell apoptosis, specifically that induced by HIV.

\section{Mechanism of HIV protease inhibitors apoptosis inhibition}

The mechanism of HIV protease inhibitor-mediated apoptosis inhibition is being actively investigated (Table 1). HIV PIs inhibit the proteolytic activity of HIV viral protease, therefore, it has been postulated that they might have a similar effect on other cellular proteases. Together with the observations that HIV PIs block Fas-mediated apoptosis, many investigators hypothesize that HIV PIs may inhibit the caspase family members and block apoptosis. Although this is an attractive model, caspases are cystine proteases and HIV protease is an aspartyle protease. Several groups have investigated the direct effect of HIV PIs on intracellular caspase activity. When recombinant caspases- $1,-3,-6,-7$, or -8 were incubated with a fluorogenic tetrapeptide substrate for each caspase in the presence of absence of nelfinavir, the HIV PI inhibited HIV protease cleavage of $\mathrm{gag} / \mathrm{pol}$, but did not inhibit the activity of any of the caspases [17]. These results were expanded in a study that demonstrated that caspases-3, -6 , and -8 activity was not inhibited by indinavir in U937 cells at drug concentrations that effectively inhibited U937 apoptosis [18]. Nelfinavir did not block activation of caspases-1, $-3,-4,-5,-9$, and -8 in lysates of Jurkat $\mathrm{T}$ cells undergoing Fas-mediated apoptosis [19].

In addition to caspases, the calpain proteases have been considered as a possible site for HIV PIs to mediate apoptosis. Calpains are $\mathrm{Ca}^{+}$-dependent cysteine proteases reported to be involved in several models of apoptosis, including U937 cells, but are not absolutely required for apoptosis [20]. Because HIV PIs are designed to inhibit the HIV cysteine protease, they may influence cellular apoptosis by blocking calpain activation and function. Ghibelli et al. demonstrated in a U937 model of apoptosis that indinavir and ritonavir directly inhibit apoptosis in cell systems where calpains are activated and block m-calpain activation [18]. Other investigators have demonstrated that ritonavir competitively inhibited activity of both $\mathrm{m}-$ and $-\mu$ calpain isoforms in PC12 cells [21]. Other investigators could not confirm this observation. These authors postulate that concentrations of ritonavir close to the maximum solubility of the drug, which was used in previous reports, may have artificially altered the results [22]. A calpain inhibitor could have significant therapeutic implications beyond HIV apoptosis, including neurodegenerative diseases; however, the evidence remains unclear whether HIV PIs have a significant effect on calpain activity.

An alternative model suggests that HIV protease inhibitors alter the expression of apoptotic regulatory proteins. Although early studies reported a change in Fas expression after PI treatment, subsequent work did not show a

Table 1 Possible methods for HIV protease inhibitor regulating apoptosis

\begin{tabular}{lc}
\hline Theoretical site of action & Support \\
\hline Caspase activity & HIV PIs block Fas and TNF mediated apoptosis \\
& HIV PIs do not block caspase-1, $-3,-4,-5,-6,-7,-8$, or -9 activity in \\
cell models & HIV PIs inhibit apoptosis in cell systems where calpains are activated \\
Calpain activity & HIV PIs blocked m-calpain activation in U937 cells \\
& HIV PIs inhibited activity of both m- and - $\mu$ calpain isoforms in PC12 \\
& cells \\
& HIV PI did not inhibit m- or $\mu$-calpain hydrolysis or activation at lower, \\
physiologic, concentrations & \\
Apoptosis regulatory proteins & No change in Fas protein levels after HIV PI treatment \\
& No change in RNA levels of Fas, Fas L, and TNF after HIV PI treatment \\
No change in Bcl-2, Bax, and Bcl-X $\mathrm{L}_{\mathrm{L}}$ after HIV PI treatment & HIV PIs maintain mitochondrial membrane integrity after apoptosis \\
Mitochondrial transmembrane potential & stimuli \\
HIV PIs prevent cytochrome $c$ release from mitochondria after & apoptosis stimuli \\
ANT (adenine nucleotide translocator) necessary for HIV PI to block & mitochondria mediated apoptosis
\end{tabular}


change in Fas levels with HIV PI therapy [14, 23-25]. Bone marrow progenitor cells from HIV-infected individuals incubated with ritonavir and indinavir showed no change in RNA levels of Fas, or Fas L. Two reports have specifically investigated the levels of intracellular apoptotic regulatory proteins with and without HIV PI treatment. Protein levels of $\mathrm{Bcl}_{2}, \mathrm{Bax}$, and $\mathrm{Bcl}-\mathrm{X}_{\mathrm{L}}$ were evaluated by flow cytometry, and were unchanged after PI administration [17, 26]. Therefore, HIV PIs can block Fas- and TNF-mediated apoptosis; this effect does not appear to be due to changes in intra- or extracellular apoptotic regulatory protein levels [14-16].

HIV PIs may also block apoptosis at the level of the mitochondria by disrupting the transmitochondrial membrane potential. The mitochondrial transmembrane potential occurs from an asymmetric distribution of ions on both sides of the inner mitochondrial membrane that is maintained by the mitochrondrial permeability transition pore complex (PTPC). After an apoptotic signal, the PTPC opens, disrupts the membrane potential, and releases apoptogenic factors, including cytochrome $c$ and procaspase-9. Thus, the mitochondria serves as a regulatory checkpoint of apoptotic signaling. Many regulatory proteins, including $\mathrm{Bcl}_{2}$ family members and IAPs, interact at the level of the mitochondria to alter apoptosis. In the first report of the effects of HIV PI on mitochondrial integrity, Jurkat cell Fas-mediated apoptosis was inhibited with $10 \mu \mathrm{M}$ of nelfinavir, a dose that would simulate physiologic levels if taken clinically. Nelfinavir treated cells maintained intact mitochondrial transmembrane potential, as determined by DioC6 staining, a lipophilic dye that stains the mitochondria [17]. The authors also reported that $10 \mu \mathrm{M}$ of nelfinavir inhibited cytochrome $c$ release during Fas-induced apoptosis. The HIV accessory molecule, Vpr, caused PTPC opening and loss of transmitochondrial potential when added directly to mitochondria. Nelfinavir pretreatment of Jurkat cells prevented Vpr-induced DioC6 release from the mitochondria and cell death. Other groups have confirmed that HIV protease inhibitors block mitochondrial transmembrane potential loss in multiple models of apoptosis [16, 17, 26-30]. In a recent report, Weaver et al. investigated how HIV PIs influence mitochondrial integrity by using yeast models. Wild type or yeast deficient in voltage-dependent anion channel (VDAC) or adenine nucleotide translocator (ANT) isoforms, two components of the PTPC, were treated with $\mathrm{Vpr}$ or $\mathrm{H}_{2} \mathrm{O}_{2}$ which induce mitochondrial apoptosis. Apoptosis only occurred after $\mathrm{Vpr}$ or $\mathrm{H}_{2} \mathrm{O}_{2}$ treatment when ANT was present. Furthermore, when Jurkat cells were pretreated with nelfinavir and then an agonist for VDAC, there was no inhibition of mitochondrial potential loss or apoptosis. However, $10 \mu \mathrm{M}$ of nelfinavir blocked ANT agonist-induced mitochondrial transmembrane potential loss and apoptosis [31]. Lastly, proteoliposomes reconstituted with ANT, which release a fluorescent dye after pore opening, did not result in pore opening when pretreated with nelfinavir before the ANT agonist [31].

In summary, there are several theories regarding the mechanism by which HIV PIs inhibit cellular apoptosis. The most data suggest that HIV PIs block apoptosis by maintaining mitochondrial integrity, likely by HIV PIs preventing pore function of the adenine nucleotide translocator subunit of the mitochondrial permeability transition pore complex (Fig. 1).

\section{HIV protease inhibitor anti-apoptotic properties during non-HIV disease states}

The novel anti-apoptotic properties of HIV PIs are being evaluated in preclinical studies as a potential therapy for disease states associated with increased levels of apoptosis such as sepsis, the leading cause of death in critically ill patients. The original description of in vivo use of HIV PIs for nonHIV related disease was in a mouse sepsis model. Sepsis is the leading cause of death in critically ill patients. Despite advances in supportive care, mortality from sepsis remains high. Animal studies demonstrate that sepsis results in extensive lymphocyte apoptosis, as well as intestinal epithelial cell apoptosis $[32,33]$. These findings have been confirmed during autopsy studies in humans who died of sepsis [34, 35]. In a mouse model of sepsis, created by cecal ligation and perforation, mice pretreated with HIV PIs had improved survival and reduced lymphocyte apoptosis [36]. The HIV PI treated mice had an increase in the Th1 cytokine TNF $\alpha$ and a reduction in the TH 2 cytokines IL- 6 and IL-10. It appears the beneficial effect of PI treatment was due to reduced lymphocyte apoptosis because lymphocyte deficient Rag 1 -/- mice had no benefit from HIV PI treatment. Follow-up work by Weaver et al. investigated the effect of HIV PIs during Staphylococcal enterotoxin B+DGal-induced shock. There was a $60 \%$ improvement in 24 -h survival in mice pretreated with HIV PIs than those treated with vehicle control. In addition, the authors also demonstrated that HIV PI pretreatment reduced mouse death from Fas-induced fatal hepatitis and middle cerebral artery occlusion-induced stroke [31].

\section{HIV protease inhibitors and stroke}

Cerebral ischemia or stroke occurs when blood flow (and thus oxygen) to the brain is reduced through hemorrhage or clot-induced occlusion of blood vessels. The only therapy with proven clinical benefit is thrombolysis requiring administration of tissue plasminogen activator within $3 \mathrm{~h}$ of the onset of ischemic attack and/or oral aspirin within the first $48 \mathrm{~h}$ after stroke onset [37]. Although the pathophysiology 


\section{HIV Protease Inhibitors Block at the level of the ANT}

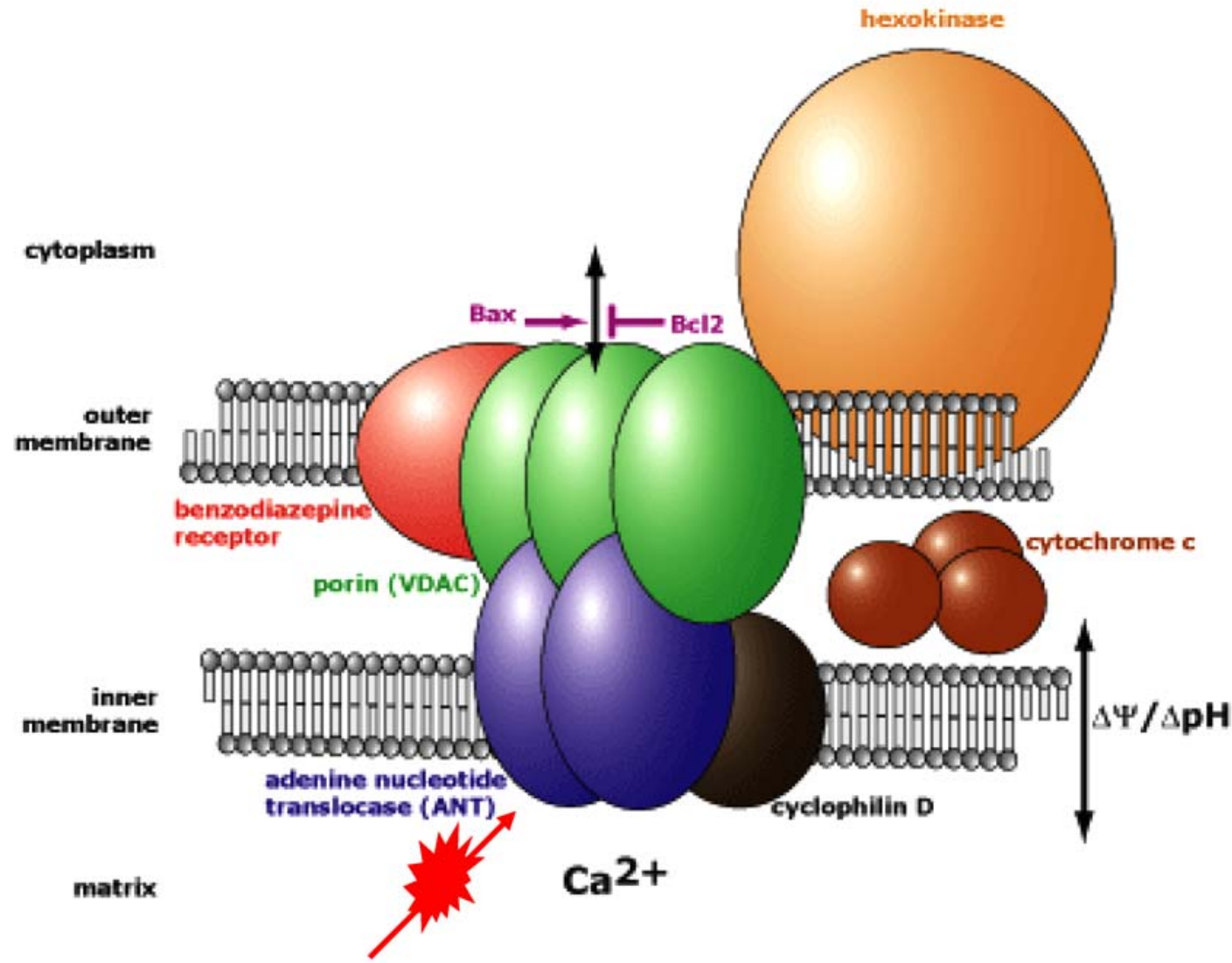

\section{HIV Protease Inhibitors}

Fig. 1 HIV protease inhibitors block cellular apoptosis at the level of the adenine nucleotide translocase (ANT) in the mitochondrial pore complex. The figure has been adapted from the Mitosciences Inc. website

of stroke damage extends well beyond this time frame, no clinical intervention capable of targeting discrete molecular mechanisms of cell death has been shown to effectively protect neurons from subsequent neuronal loss. Elucidation of the complex and multiple cell death pathways initiated by ischemia has made it increasingly apparent that a broader therapeutic approach is required [37-40]. These findings are supported by correlative clinical evidence of neuroprotective activity in other central nervous system disorders following treatment with PIs [41, 42].

Stroke elicits rapid necrotic and excitotoxic mechanisms as well as delayed apoptotic-like responses. Cell death is initiated once core tissue is deprived of oxygen-rich blood. Immediately following vessel occlusion, cell viability is lost, in part, by a reduction in ATP levels resulting in an efflux of $\mathrm{K}^{+}$ions from compromised neurons and glia and an influx of
$\mathrm{Na}^{+}, \mathrm{Cl}^{-}$, and $\mathrm{Ca}^{2+}$ ions. Neuronal depolarization resulting from a loss of calcium homeostasis is further exacerbated by the sustained release of glutamate and reduction in extracellular $\mathrm{pH}$ leading to excitotoxicity. Production of free fatty acids and oxyradicals are elevated, triggering oxidative remodeling of membrane lipids, impaired glial homeostatic functions and enhanced inflammatory cell activation [40, 43, 44].

Studies using primary hippocampal neurons indicate that HIV PIs can protect neurons from cell death triggered by membrane lipid remodeling associated with oxidative stress. Ritonavir has been shown to inhibit neuronal injury triggered in vitro by 4-hydroxynonenal, a lipid-soluble aldehydic product of membrane peroxidation that impairs $\mathrm{Na}^{+}, \mathrm{K}^{+}$, and ATPase activity [45]. In vivo, administration of nelfinavir and ritonavir prior to ischemic insult effectively reduces infarct 
size in mice following middle cerebral artery occlusion, resulting in functional recovery of ischemic neurons [31].

Mechanistic assessment suggests that HIV PIs act to reduce delayed neuronal ischemic death triggered also through mitochondrial-dependent pathways. Neurons located at the periphery of the necrotic core (dubbed the penumbra) are spared acute ischemic injury. Damage is not evident until hours, days, and weeks following reperfusion when cells begin to exhibit many of the morphological and biochemical characteristics of apoptosis [45]. Death is likely triggered during ischemia/reperfusion by downstream release of internal calcium stores from endoplasmic reticulum and mitochondria [43]. Additional death pathways are subsequently initiated by a complex cross-talk between extrinsic death receptor mediated-induction and intrinsic mitochondrialdependent pathways. Briefly, extrinsic induction involves binding of apoptogens, such as Fas ligand and tumor necrosis factor $\alpha(\mathrm{TNF} \alpha)$, to death domain-containing receptors inducing oligomerization and recruitment of the adapter proteins FADD and TRADD [46]. Complex formation between adapter proteins, receptor death domains, and procaspases accelerates the autocatalytic activation of procaspase- $8,-10$, and -2 . Once cleaved, these initiator caspases can cleave and activate executioner caspases- $3,-6$, and -7 responsible for the regulated disassembly of cellular proteins characteristic of apoptosis [47]. Intrinsic cell death is dictated by mitochondrial function. Release of reactive oxygen species following ischemic reperfusion triggers release of cytochrome $c$ and ATP from compromised mitochondria into the cytosol, often with concomitant loss of loss of $\Delta y_{\mathrm{m}}$. Activation of caspase 9 occurs with formation of the apoptosome composed of APAF-1, cytochrome $c$, ATP, and procaspase 9 resulting in downstream activation of caspase- $3,-6,-7$ [43, 46].

HIV PIs act at multiple mitochondrial branch points of these overlapping apoptotic cascades to reduce delayed neuronal death following ischemic insult. Pretreatment with nelfinavir prior to apoptotic challenge inhibits the pore function of the adenine nucleotide transporter, thereby reducing the loss of loss of $\Delta y_{\mathrm{m}}$, apoptosome formation, and downstream caspase activation [31]. Because HIV PIs inhibit the downstream mitochondrial events elicited by caspase 8-mediaed cleavage of Bid, downstream events of the extrinsic pathway are also reduced [31].

Recent unpublished data from our laboratories indicate that this protection may also be of potential clinical benefit even if administered after the ischemic event-a key requirement for successful adjuvant therapies. Loss of CA1 pyramidal neurons in the hippocampus is a hallmark of global ischemia. Ischemic hippocampal injury can be experimentally designed by the two-vessel occlusion rodent model of global ischemia in which both internal carotid arteries are transiently ligated. As demonstrated in Fig. 2, we observed significant sparing of CA1 pyramidal neurons whether ani-
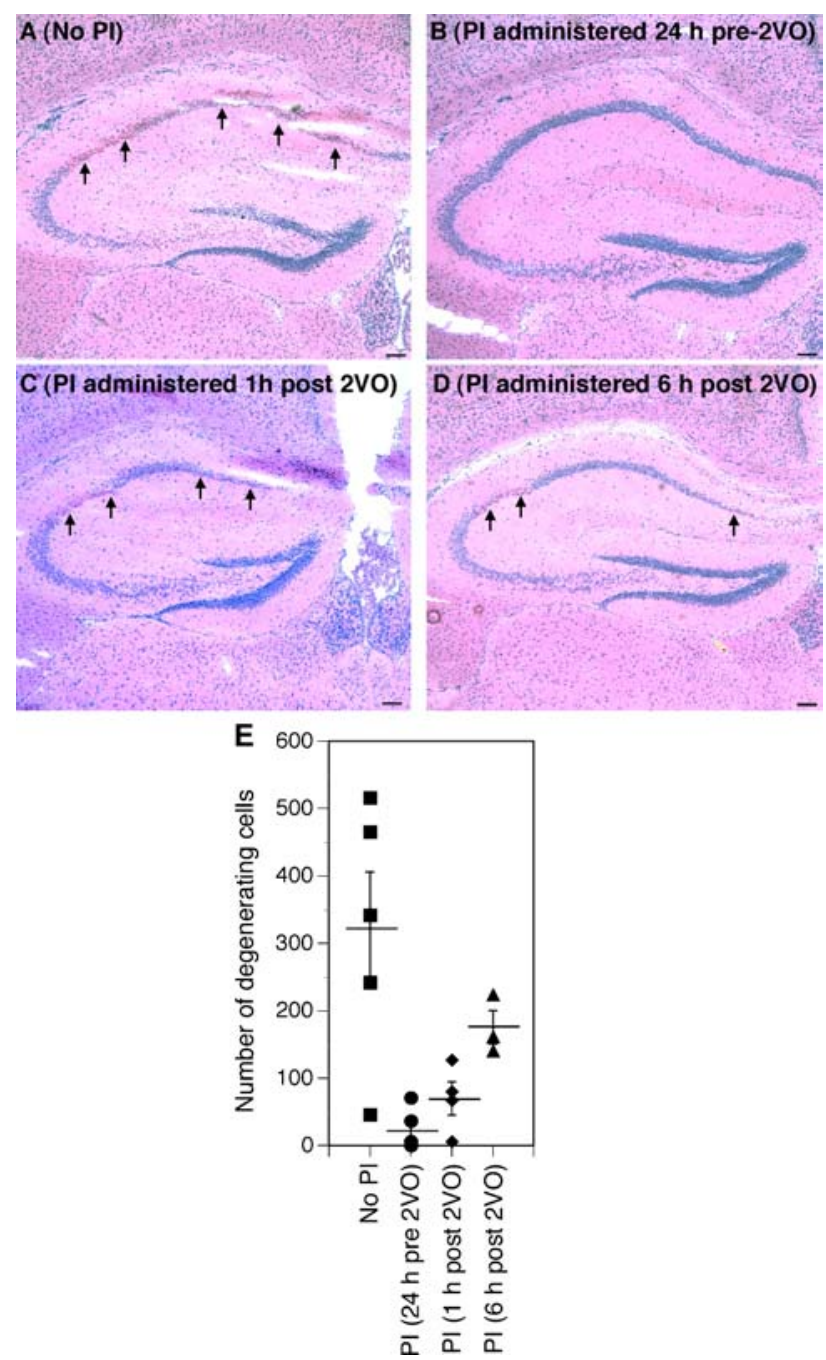

Fig. 2 Effects of nelfinavir and ritonavir on ischemic hippocampal damage induced by two vessel carotid occlusion. Mice subjected to two vessel occlusion (A) were compared to animals that received three oral gavages of either nelfinavir or ritonavir $8 \mathrm{~h}$ apart starting $24 \mathrm{~h}$ before (B), $1 \mathrm{~h}$ after (C), or $6 \mathrm{~h}$ after (D) carotid occlusion. Arrows indicate areas of ischemic damage. Viable cell number in the CA1 was counted $48 \mathrm{~h}$ after ischemic onset in hemotoxylin and eosin-stained sections (E). Data represent the average number of viable cells in both hippocampi and are expressed per animal. The mean number of viable cells per condition \pm standard error of measurement is indicated. Neurodegeneration was completely attenuated by pre-treatment with PIs and partially attenuated by administration of PIs following carotid occlusion. Scale bars, $100 \mu \mathrm{m}$

mals received nelfinavir boosted by ritonavir prior to or immediately after $2 \mathrm{VO}$ surgery (Fig. 2). Neuroprotection was still evident, albeit at reduced efficacy, when nelfinavir and ritonavir were administered up to $6 \mathrm{~h}$ after two-vessel carotid occlusion (Fig. 2), suggesting a wider therapeutic window than previously anticipated [31].

While promising, mechanistic assessment of HIV PIs in stroke management is still in early days. Although HIV PIs appear to inhibit apoptosis in multiple organs at 
concentrations comparable to that seen in the plasma following clinical application (reviewed in [48]), these same compounds trigger cell death at higher concentrations [49-51]. Moreover, chronic administration, as part of HIV treatment, has been associated with an enhanced risk of ischemia in both central and peripheral tissue. Chronic HIV PI treatment can elicit hyperlipidemia, accelerating atherosclerosis and represent a potential increased risk for myocardial infarction [52-54]. As such, it is unlikely that HIV PIs can be used prophylactically to prevent stroke damage in susceptible individuals. However, the promise of transient administration is promising. The impact of acute administration of HIV PIs following stroke requires a thorough evaluation of the therapeutic window open to mitochondrial manipulation and is essential to validate the promise of HIV PIs as a potential adjuvant strategy to promote neuronal survival following ischemia.

\section{HIV protease inhibitors and pancreatitis}

Accelerated apoptosis also contributes to cellular injury during acute pancreatitis. Our group investigated whether treatment with the HIV protease inhibitors nelfinavir/ritonavir would reduce the severity of pancreatitis using a mouse caerulein-induced pancreatitis model. Ritonavir was used to increase nelfinavir drug levels to a therapeutic level in the mice. Mice treated with nelfinavir/ritonavir before caerulein induced pancreatitis had reduced serum amylase levels and less acinar injury of the pancreas on histologic review, compared to mice pretreated with vehicle control.

\section{HIV protease inhibitors and T cell production}

The anti-apoptotic effects of HIV protease inhibitors may have indirect beneficial clinical effects as well. In a recent report by Graham et al., five out of seven HIV-negative patients treated with an HIV protease inhibitor containing HAART regimen for a needle-stick exposure experienced a 3-log increase in thymic-derived naïve $\mathrm{T}$ cells in the peripheral blood. The increase in naïve $\mathrm{T}$ cells, as determined by $\mathrm{T}$ cell receptor recombination excision circle levels (TREC), occurred after four weeks of therapy, suggesting, in yet another setting, that HIV PI can have beneficial immunemodulatory effects [55].

\section{Paradoxical pro-apoptotic effect of HIV protease inhibitors}

Under certain conditions HIV PIs may be pro-apoptotic as well. At increased doses of HIV PI, it is possible that the drug may interfere with cellular activation signals that result in transformation and protect the tumor cell from apoptosis. High concentrations of ritonavir $(10-50 \mu \mathrm{M})$ inhibit the proliferation of murine and human tumor cell lines. DNA laddering demonstrated that $15-30 \mu \mathrm{M}$ of ritonavir induced apoptosis in the same cell lines [56]. Of note, the concentrations of ritonavir used were over 15 times what most adults achieve at FDA-approved doses and at which the drug blocks apoptosis. This led to further work developing the pro-apoptotic effects of HIV PIs. Adult T-cell leukemia (ATL) is an aggressive malignancy associated with human T-cell leukemia virus (HTLV) and is very resistant to conventional chemotherapy. When ATL cells were incubated with $20-40 \mu \mathrm{M}$ of ritonavir, there was a five-fold increase in spontaneous apoptosis, resulting in a similar decrease in tumor cell survival [57]. In ATL cell lines and primary ATL cells $40 \mu \mathrm{M}$ of ritonavir inhibited transcriptional activation of NF- $\kappa \mathrm{B}$. In addition, HIV PIs inhibited the expression of the targets of NF- $\kappa \mathrm{B}, \mathrm{Bcl}-\mathrm{X}_{\mathrm{L}}$, survivin, c-Myc, and cyclin D2 [57].

There is also evidence that HIV protease inhibitors are pro-apoptotic in models of solid tumors. Freshly isolated multiple myeloma cells from patients under went apoptosis after incubation with $40-50 \mu \mathrm{M}$ of ritonavir, saquinavir, and nelfinavir. This was associated with a decrease in the anti-apoptotic protein Mcl-1, and blocked IL-6 phosphorylation of ERK 1/2 and STAT 3 [58]. Different tumor types may have different mechanisms by which HIV PIs inhibit tumor growth and promote apoptosis. Ritonavir used at $20 \mu \mathrm{M}$ in solid and hematologic tumor models caused an increase in the cellular concentrations of the antiproliferative and pro-apoptotic proteasome substrate cdk inhibitor, p21. This was associated with a block in proteolytic degradation [56], consistent with an earlier report that HIV PI impact proteasome activity [59]. Accumulation of intracellular p21 resulted in cell-cycle arrest in $\mathrm{G}_{1}$ phase and subsequent apoptosis in ritonavir treated tumor cells.

HIV protease inhibitors are well-tolerated drugs with enticing possibilities for future use to alter apoptosis in many human disease states. Clinical trials of HIV PIs in disease states other than HIV are already underway investigating the in vivo effects on human cellular apoptosis (NCT00346619 and NCT00233948). Furthermore, now that the target for how HIV PIs block cellular apoptosis has been identified as the mitochondrial pore protein ANT, physiochemical optimization can be performed. Lastly, the mechanism by which high concentrations of HIV PIs induce apoptosis in transformed tumor models needs to be further clarified. Studies are needed to determine whether the HIV PIs alter proteins that modulate cellular proliferation or apoptosis, and whether there is a threshold to the pro-apoptotic effects. 
Acknowledgments SRV is supported by a grant from the Mayo Program in Translational Immunovirology and Biodefense and a Robert and Arlene Kogod Program on Aging Award from the Mayo Foundation. ADB is supported by NIH grants RO1-AI62261 and RO1AI40384, and a Burroughs Wellcome Award ID\#1005160.

\section{References}

1. Collier AC, Coombs RW, Schoenfeld DA et al (1996) Treatment of human immunodeficiency virus infection with saquinavir, zidovudine, and zalcitabine: AIDS Clinical Trials Group. N Engl J Med 334(16):1011-1017

2. Kravcik S, Magill A, Sanghvi B et al (2001) Comparative CD4 T-cell responses of reverse transcriptase inhibitor therapy with or without nelfinavir matched for viral exposure. HIV Clin Trials 2(2):160-170

3. Deeks SG, Grant RM. (1999) Sustained CD4 responses after virological failure of protease inhibitor-containing therapy. Antivir Ther 4(Suppl 3):7-11

4. Albrecht MA, Bosch RJ, Hammer SM et al (2001) Nelfinavir, efavirenz, or both after the failure of nucleoside treatment of HIV infection. N Engl J Med 345(6):398-407

5. Staszewski S, Morales-Ramirez J, Tashima KT et al (1999) Efavirenz plus zidovudine and lamivudine, efavirenz plus indinavir, and indinavir plus zidovudine and lamivudine in the treatment of HIV-1 infection in adults. Study 006 Team. N Engl J Med 341(25):1865-1873

6. Owen C, Kazim F, Badley AD (2004) Effect on CD4 T-cell count of replacing protease inhibitors in patients with successful HIV suppression: a meta-analysis. Aids 18(4):693-695

7. Arribas JR, Pulido F, Delgado R et al (2005) Lopinavir/ritonavir as single-drug therapy for maintenance of HIV-1 viral suppression: 48-week results of a randomized, controlled, open-label, proof-ofconcept pilot clinical trial (OK Study). J Acquir Immune Defic Syndr 40(3):280-287

8. Fethi T, Asma J, Amine SM et al (2005) Effects on immunological and virological outcome of patients using one protease inhibitor or one non-nucleoside reverse transcriptase inhibitor in a triple antiretroviral therapy: normal clinical practice versus clinical trial findings. Curr HIV Res 3(3):271-276

9. Badley AD, Dockrell DH, Algeciras A et al (1998) in vivo analysis of Fas/FasL interactions in HIV-infected patients. J Clin Invest 102(1):79-87

10. Landay AL, Spritzler J, Kessler H et al (2003) Immune reconstitution is comparable in antiretroviral-naive subjects after 1 year of successful therapy with a nucleoside reverse-transcriptase inhibitor- or protease inhibitor-containing antiretroviral regimen. J Infect Dis 188(10):1444-1454

11. Badley AD, Parato K, Cameron DW et al (1999) Dynamic correlation of apoptosis and immune activation during treatment of HIV infection. Cell Death Differ 6(5):420-432

12. Phenix BN, Angel JB, Mandy F et al (2000) Decreased HIVassociated $\mathrm{T}$ cell apoptosis by HIV protease inhibitors. AIDS Res Hum Retroviruses 16(6):559-567

13. Sloand EM, Maciejewski J, Kumar P, Kim S, Chaudhuri A, Young N. (2000) Protease inhibitors stimulate hematopoiesis and decrease apoptosis and ICE expression in CD34(+) cells. Blood 96(8):2735-2739

14. Estaquier J, Lelievre JD, Petit F et al (2002) Effects of antiretroviral drugs on human immunodeficiency virus type 1-induced CD4(+) T-cell death. J Virol 76(12):5966-5973

15. Wolf T, Findhammer S, Nolte B, Helm EB, Brodt HR. (2003) Inhibition of TNF-alpha mediated cell death by HIV-1 specific protease inhibitors. Eur J Med Res 8(1):17-24
16. Matarrese P, Tinari A, Gambardella L et al (2005) HIV protease inhibitors prevent mitochondrial hyperpolarization and redox imbalance and decrease endogenous uncoupler protein- 2 expression in gp 120-activated human T lymphocytes. Antivir Ther 10(Suppl 2):M29-M45

17. Phenix BN, Lum JJ, Nie Z, Sanchez-Dardon J, Badley AD. (2001) Antiapoptotic mechanism of HIV protease inhibitors: preventing mitochondrial transmembrane potential loss. Blood 98(4):10781085

18. Ghibelli L, Mengoni F, Lichtner M et al (2003) Anti-apoptotic effect of HIV protease inhibitors via direct inhibition of calpain. Biochem Pharmacol 66(8):1505-1512

19. Chavan S, Kodoth S, Pahwa R, Pahwa S (2001) The HIV protease inhibitor Indinavir inhibits cell-cycle progression in vitro in lymphocytes of HIV-infected and uninfected individuals. Blood 98(2):383-389

20. Spinedi A, Oliverio S, Di Sano F, Piacentini M (1998) Calpain involvement in calphostin C-induced apoptosis. Biochem Pharmacol 56(11):1489-1492

21. Wan W, DePetrillo PB (2002) Ritonavir inhibition of calciumactivated neutral proteases. Biochem Pharmacol 63(8):1481-1484

22. Cuerrier D, Nie Z, Badley AD, Davies PL (2005) Ritonavir does not inhibit calpain in vitro. Biochem Biophys Res Commun 327(1):208-211

23. Sloand EM, Kumar PN, Kim S, Chaudhuri A, Weichold FF, Young NS (1999) Human immunodeficiency virus type 1 protease inhibitor modulates activation of peripheral blood CD4(+) T cells and decreases their susceptibility to apoptosis in vitro and in vivo. Blood 94(3):1021-1027

24. Lu W, Andrieu JM (2000) HIV protease inhibitors restore impaired T-cell proliferative response in vivo and in vitro: a viral-suppression-independent mechanism. Blood 96(1):250258

25. Isgro A, Aiuti A, Mezzaroma I et al (2005) HIV type 1 protease inhibitors enhance bone marrow progenitor cell activity in normal subjects and in HIV type 1-infected patients. AIDS Res Hum Retroviruses 21(1):51-57

26. Matarrese P, Gambardella L, Cassone A, Vella S, Cauda R, Malorni W (2003) Mitochondrial membrane hyperpolarization hijacks activated $\mathrm{T}$ lymphocytes toward the apoptotic-prone phenotype: homeostatic mechanisms of HIV protease inhibitors. J Immunol 170(12):6006-6015

27. Weichold FF, Bryant JL, Pati S, Barabitskaya O, Gallo RC, Reitz Jr MS (1999) HIV-1 protease inhibitor ritonavir modulates susceptibility to apoptosis of uninfected T cells. J Hum Virol 2(5):261-269

28. Badley AD, Roumier T, Lum JJ, Kroemer G (2003) Mitochondrion-mediated apoptosis in HIV-1 infection. Trends Pharmacol Sci 24(6):298-305

29. Garg H, Blumenthal R (2006) HIV gp41-induced apoptosis is mediated by caspase-3-dependent mitochondrial depolarization, which is inhibited by HIV protease inhibitor nelfinavir. J Leukoc Biol 79(2):351-362

30. Miro O, Villarroya J, Garrabou G et al (2005) in vivo effects of highly active antiretroviral therapies containing the protease inhibitor nelfinavir on mitochondrially driven apoptosis. Antivir Ther 10(8):945-951

31. Weaver JG, Tarze A, Moffat TC et al (2005) Inhibition of adenine nucleotide translocator pore function and protection against apoptosis in vivo by an HIV protease inhibitor. J Clin Invest 115(7): 1828-1838

32. Ayala A, Herdon CD, Lehman DL, Ayala CA, Chaudry IH (1996) Differential induction of apoptosis in lymphoid tissues during sepsis: variation in onset, frequency, and the nature of the mediators. Blood 87(10):4261-4275

33. Hotchkiss RS, Swanson PE, Cobb JP, Jacobson A, Buchman TG, Karl IE (1997) Apoptosis in lymphoid and parenchymal cells dur- 
ing sepsis: findings in normal and T- and B-cell-deficient mice. Crit Care Med 25(8):1298-1307

34. Husain KD, Coopersmith CM (2003) Role of intestinal epithelial apoptosis in survival. Curr Opin Crit Care 9(2):159-163

35. Coopersmith CM, Chang KC, Swanson PE et al (2002) Overexpression of $\mathrm{Bcl}-2$ in the intestinal epithelium improves survival in septic mice. Crit Care Med 30(1):195-201

36. Weaver JG, Rouse MS, Steckelberg JM, Badley AD (2004) Improved survival in experimental sepsis with an orally administered inhibitor of apoptosis. Faseb J 18(11):1185-1191

37. Ly JV, Zavala JA, Donnan GA (2006) Neuroprotection and thrombolysis: combination therapy in acute ischaemic stroke. Expert Opin Pharmacother 7(12):1571-1581

38. Lee JM, Zipfel GJ, Choi DW (1999) The changing landscape of ischaemic brain injury mechanisms. Nature 399(6738 Suppl):A7A14

39. Reed JC (2001) Apoptosis-regulating proteins as targets for drug discovery. Trends Mol Med 7(7):314-319

40. Lo EH, Moskowitz MA, Jacobs TP (2005) Exciting, radical, suicidal: how brain cells die after stroke. Stroke 36(2):189-192

41. Rosenfeldt V, Valerius NH, Paerregaard A (2000) Regression of HIV-associated progressive encephalopathy of childhood during HAART. Scand J Infect Dis 32(5):571-574

42. MacGowan DJ, Scelsa SN, Waldron M (2001) An ALS-like syndrome with new HIV infection and complete response to antiretroviral therapy. Neurology 57(6):1094-1097

43. Lopez-Neblina F, Toledo AH, Toledo-Pereyra LH (2005) Molecular biology of apoptosis in ischemia and reperfusion. J Invest Surg 18(6):335-350

44. MacDonald JF, Xiong ZG, Jackson MF (2006) Paradox of Ca2+ signaling, cell death and stroke. Trends Neurosci 29(2):75-81

45. Wan W, DePetrillo PB (2002) Ritonavir protects hippocampal neurons against oxidative stress-induced apoptosis. Neurotoxicology 23(3):301-306

46. Ferrer I, Planas AM (2003) Signaling of cell death and cell survival following focal cerebral ischemia: life and death struggle in the penumbra. J Neuropathol Exp Neurol 62(4):329-339

47. Zheng Z, Zhao H, Steinberg GK, Yenari MA (2003) Cellular and molecular events underlying ischemia-induced neuronal apoptosis. Drug News Perspect 16(8):497-503

48. Phenix BN, Cooper C, Owen C, Badley AD (2002) Modulation of apoptosis by HIV protease inhibitors. Apoptosis 7(4):295-312
49. Pajonk F, Himmelsbach J, Riess K, Sommer A, McBride WH (2002) The human immunodeficiency virus (HIV)-1 protease inhibitor saquinavir inhibits proteasome function and causes apoptosis and radiosensitization in non-HIV-associated human cancer cells. Cancer Res 62(18):5230-5235

50. Zhong DS, Lu XH, Conklin BS et al (2002) HIV protease inhibitor ritonavir induces cytotoxicity of human endothelial cells. Arterioscler Thromb Vasc Biol 22(10):1560-1566

51. Bode H, Lenzner L, Kraemer OH et al (2005) The HIV protease inhibitors saquinavir, ritonavir, and nelfinavir induce apoptosis and decrease barrier function in human intestinal epithelial cells. Antivir Ther 10(5):645-655

52. Penzak SR, Chuck SK (2002) Management of protease inhibitorassociated hyperlipidemia. Am J Cardiovasc Drugs 2(2):91106

53. Lai S, Lai H, Celentano DD et al (2003) Factors associated with accelerated atherosclerosis in HIV-1-infected persons treated with protease inhibitors. AIDS Patient Care STDS 17(5):211-219

54. Cohen CJ (2005) Ritonavir-boosted protease inhibitors, Part 2: cardiac implications of lipid alterations. AIDS Read 15(10):528532

55. Graham DB, Bell MP, Huntoon CJ et al (2005) Increased thymic output in HIV-negative patients after antiretroviral therapy. Aids 19(14):1467-1472

56. Gaedicke S, Firat-Geier E, Constantiniu O et al (2002) Antitumor effect of the human immunodeficiency virus protease inhibitor ritonavir: induction of tumor-cell apoptosis associated with perturbation of proteasomal proteolysis. Cancer Res 62(23):69016908

57. Dewan MZ, Uchihara JN, Terashima K et al (2006) Efficient intervention of growth and infiltration of primary adult T-cell leukemia cells by an HIV protease inhibitor, ritonavir. Blood 107(2):716724

58. Ikezoe T, Saito T, Bandobashi K, Yang Y, Koeffler HP, Taguchi H (2004) HIV-1 protease inhibitor induces growth arrest and apoptosis of human multiple myeloma cells via inactivation of signal transducer and activator of transcription 3 and extracellular signalregulated kinase 1/2. Mol Cancer Ther 3(4):473-479

59. Andre P, Groettrup M, Klenerman P et al (1998) An inhibitor of HIV-1 protease modulates proteasome activity, antigen presentation, and T cell responses. Proc Natl Acad Sci USA 95(22):1312013124 